\title{
The real genus of the alternating groups
}

\author{
José Javier Etayo Gordejuela and Ernesto Martínez
}

\begin{abstract}
A Klein surface with boundary of algebraic genus $\mathfrak{p} \geq 2$, has at most $12(\mathfrak{p}-1)$ automorphisms. The groups attaining this upper bound are called $M^{*}$-groups, and the corresponding surfaces are said to have maximal symmetry. The $M^{*}$-groups are characterized by a partial presentation by generators and relators.

The alternating groups $A_{n}$ were proved to be $M^{*}$-groups when $n \geq 168$ by M. Conder. In this work we prove that $A_{n}$ is an $M^{*}$ group if and only if $n \geq 13$ or $n=5,10$. In addition, we describe topologically the surfaces with maximal symmetry having $A_{n}$ as automorphism group, in terms of the partial presentation of the group. As an application we determine explicitly all such surfaces for $n \leq 14$.

Each finite group $G$ acts as an automorphism group of several Klein surfaces. The minimal genus of these surfaces is called the real genus of the group, $\rho(G)$. If $G$ is an $M^{*}$-group then $\rho(G)=\frac{o(G)}{12}+1$. We end our work by calculating the real genus of the alternating groups which are not $M^{*}$-groups.
\end{abstract}

\section{Introduction and Preliminaries}

A Klein surface is a compact surface $S$ endowed with a dianalytic structure. The surface $S$ can be orientable or not, with or without boundary. Klein surfaces with algebraic genus $\mathfrak{p} \geq 2$ have a finite group of automorphisms whose order has an upper bound in terms of $\mathfrak{p}$. More precisely, the orientable surfaces without boundary are called Riemann surfaces, and such a surface of genus $\mathfrak{p}$ has at most $84(\mathfrak{p}-1)$ automorphisms. If this bound is attained the group of automorphisms is said to be a Hurwitz group. In case the surface is non-orientable without boundary the corresponding bound is $84(\mathfrak{p}-2)$ and

2000 Mathematics Subject Classification: Primary: 20F05. Secondary: 30F10.

Keywords: Alternating groups, real genus, $M^{*}$-groups, bordered Klein surfaces. 
the group is an $H^{*}$-group. Finally for surfaces with boundary of algebraic genus $\mathfrak{p}$ the automorphism group has order at most $12(\mathfrak{p}-1)$ and then it is called an $M^{*}$-group.

In this work we determine which alternating groups $A_{n}$ are $M^{*}$-groups. Marston Conder proved in the early 80's that all alternating groups $A_{n}$ are $M^{*}$-groups for $n \geq 168$, as well as for 87 values of $n<168$, starting by $n=15,28,35,42$, and so on, [3]. For small values of $n$ it is known that there are groups which are $M^{*}$-groups and others are not. The first author proved in [8] that $A_{n}$ is an $M^{*}$-group for $n=5$ (see also [13]), 10,13, and it is not for $n=6$ (see also [14]), 7,8 .

We now fill the gaps, proving that $A_{n}$ is an $M^{*}$-group for all $n \geq 13$, and $A_{5}$ and $A_{10}$ are the unique $M^{*}$-groups for $n<13$.

The $M^{*}$-groups are characterized by a partial presentation by generators and relators: a group $G$ is an $M^{*}$-group if and only if there exists a set of generators $a, b, c$, such that $a, b, c$ and $a b$ have order 2 , and $a c$ has order 3 . This presentation is closely related to the groups $G^{3, p, q}$ introduced by Coxeter in 1939, [6]. If we call $p$ the order of $b c$ and $q$ the order of $a b c$, the $M^{*}$-group $G$ is a quotient of $G^{3, p, q}$.

For later convenience we relabel the generators as follows:

$$
\begin{aligned}
X & =a c, \\
A & =a b, \\
B & =a, \\
X B & =a c a, \\
A B & =b .
\end{aligned}
$$

Then the group $G$ is generated by $X, A, B$. The order of $X$ is 3, the order of $A, B, X B$ and $A B$ is 2 , the order of $A X$ is $p$ and the order of $A B X$ is $q$. Obviously the order of $X A$ is then $p$ and the order of $X A B$ is $q$. Besides by interchanging $A$ and $A B$, we permute the roles of $p$ and $q$, and hence $G^{3, p, q} \approx G^{3, q, p}$.

So the problem of deciding if a given group is an $M^{*}$-group is reduced to finding generators $X, A, B$, satisfying the above conditions, or alternatively to prove that they do not exist.

In order to prove that the elements $X, A, B$ generate the group $A_{n}$ the main tool is the following Theorem of Jordan (1873) (see [22, Th. 13.9]).

Theorem A. Let $p$ be a prime and $G$ a primitive group of degree $n=p+k$, $k \geq 3$. If $G$ contains an element of degree and order $p$, then $G$ is alternating or symmetric. 
Since our elements $A$ and $B$ are always even permutations we need only to fulfill the condition of Theorem A to generate $A_{n}$. As a consequence of this Theorem A we have the following result from [2, Corollary 1.12]:

Corollary B. Let $g$ and $h$ be even permutations which generate a transitive group $G$. If $g$ contains a cycle of prime length $p$, where $p<n-2, p$ divides the length of no other cycle of $g$, and the p-cycle contains either a fixed point of $h$ or the points from a cycle of $h$, then $G$ is $A_{n}$.

In order to prove these conditions we take into account that the group is generated by the pair $X A, B$. For, $[X A, B]^{2}=X, X^{2}(X A)=A$, and so $<X A, B>=<X, A, B>$. We shall apply the Corollary B taking $g$ as $X A$ and $h=B$.

Alternatively, to prove that a given group is not an $M^{*}$-group, we need to check that there exists no triple of generators $X, A, B$, in above conditions. We obtained in [9] the conditions that $B$ must fulfill, and summarize them here:

Proposition C. Let $X$ and $A$ be two elements of $A_{n}$ of orders three and two, respectively. An element $B \in A_{n}$ of order two satisfies that $A B$ and $X B$ have order two if and only if:

1) B maps fixed points of $X$ onto fixed points of $X$, and fixed points of $A$ onto fixed points of $A$.

2) If $(x, y)$ and $(z, t)$ are 2 -cycles of $A$, and $B$ maps $x$ onto $z$, then $(y, t)$ is a 2-cycle of $B$. In particular, if $x$ is a fixed point of $B$, so is $y$.

3) If $(x, y, z)$ and $(t, v, w)$ are 3 -cycles of $X$, and $B$ maps $x$ onto $t$, then $(y, w)$ and $(z, v)$ are 2 -cycles of $B$. As a particular case, if $x$ is a fixed point of $B$ then $(y, z)$ is a 2-cycle of $B$.

Now we describe the different Sections on the paper. Section 2 is devoted to introducing a diagrammatic argument inspired in the construction by Conder in [2], although we use different diagrams chosen ad hoc.

In Section 3 with the groups $A_{n}$ with $13 \leq n<168$, to prove that they all are $M^{*}$-groups. The two first cases $n=13,14$, will be managed directly. For the remaining cases we use the diagrams explained in Section 2. We shall need to split the proof into twelve cases according to the residue $r$ of $n \bmod 12$. In every case the procedure is similar: we obtain the suitable generators for the first value of $n$ in that set, which we denote by $n_{0}=$ $12 t_{0}+r$, and indicate how to add $12 t$ new vertices to the diagram in such a way to assure that for each $t$, the corresponding $A_{n}$ has an adequate set of generators. 
For each $n$ we shall look for permutations $X, A$, and $B$ such that $X A$ be a product of three or four cycles, verifying that one of them, say $P$, has prime length, another one, $Q$, has even length, and the length of $P$ does not divide another length. Moreover, the cycle $P$ will contain either a fixed point of $B$ or two points paired by $B$. Hence $P$ will satisfy the conditions of the Corollary B and the corresponding $A_{n}$ will be an $M^{*}$-group.

In fact we shall prove a stronger result. For each $n=12 t+r$, we shall find several different sets of generators satisfying Corollary B. For each set the length of the cycle $P$ will be a prime number depending on $t_{0}$ and $r$.

Once this is done, in Section 4 we deal with the lower values of $n$ to prove that just $A_{5}$ and $A_{10}$ are $M^{*}$-groups for $n<13$.

Section 5 is devoted to the study of the surfaces which have these groups as automorphism group. We prove that they are topologically determined by the information we have on the group, and obtain explicitly all of them for the groups $A_{5}, A_{10}, A_{13}$ and $A_{14}$.

Finally Section 6 is written in terms of real genus. Given a group $G$, its real genus $\rho(G)$ is the minimum algebraic genus of the surfaces on which $G$ acts as automorphism group. Obviously if $G$ is an $M^{*}$-group, $\rho(G)=$ $o(G) / 12+1$. The real genus of just six alternating group remains to be determined and it is calculated in this Section.

Remark 1. Related results on the action of alternating groups on Riemann surfaces were obtained by Conder. For these surfaces the relevant point is that the group be generated by two elements of orders 2 and 3. In [5] it is seen that all but 14 alternating groups are generated by two such elements whose product has order 7 or 8 . However this fact does not imply by itself that $A_{n}$ be an $M^{*}$-group: see just the paper [5], or else [9] where $A_{21}, A_{22}$ and $A_{29}$ are discussed in detail.

\section{The diagrammatic procedure}

For each of the twelve classes according to the residue of $n$ modulo 12 , we give a starting diagram as simple as possible corresponding to the first value of $n$. In this diagram of $n$ vertices the triangles correspond to the 3 -cycles of $X$ read anticlockwise. The edges are of three types: i) their ends belong to two different triangles; ii) only one end is in a triangle; iii) both ends belong to the same triangle. In the latter type we shall indicate it in the diagrams by a curved segment (see Figure 5 below). The edges correspond to the 2-cycles of $A$. The points paired by the vertical axis of symmetry correspond to the 2-cycles of $B$. The fixed points of $X$ are the ends of 
edges (of type ii) not lying in a triangle. It is easy to see that $X, A$ and $B$ fulfill the conditions in Proposition $\mathrm{C}$ and so these permutations satisfy $(X B)^{2}=(A B)^{2}=1$.

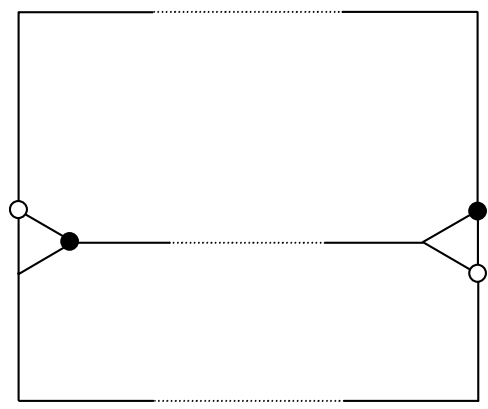

Figure 1.

Let us observe the diagram in Figure 1, where the dotted lines stand for several triangles and edges which will be seen in each case. The four corners in the picture are not vertices neither of this diagram nor of the following ones. Let us assume that the black points belong to a cycle of $X A$ and the white points belong to another different cycle.

In the upper half of the diagram we add four new triangles on the vertical lines symmetrically with respect to the vertical axis. We have three possibilities as it is seen in Figure 2.

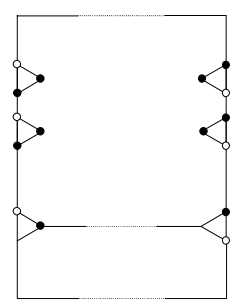

(a)

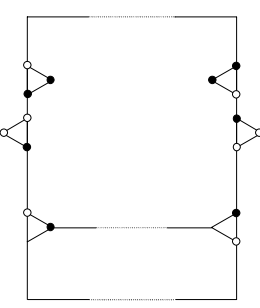

(b)

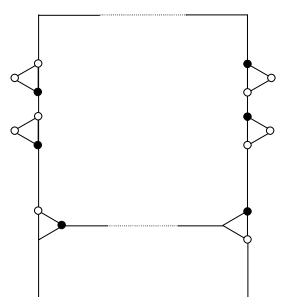

(c)

Figure 2.

We see that in case (a) the length of the "black" cycle $\mathcal{B}$ has increased in 8 , in case (b) in 6 and in the case (c) in 4. The length of the "white" cycle $\mathcal{W}$ has increased in 4,6 or 8, respectively. Let us call insider block, mixed block and outsider block, respectively, the added blocks in cases (a), (b) and (c).

Let us suppose that we have a "black" cycle with length $m_{b}$ and a "white" cycle with length $m_{w}$. If we add $x$ insider blocks, $y$ mixed blocks and $z$ outsider blocks the lengths of the new cycles $\mathcal{B}$ and $\mathcal{W}$ will become $|\mathcal{B}|=$ $m_{b}+8 x+6 y+4 z$ and $|\mathcal{W}|=m_{w}+4 x+6 y+8 z$. 
Proposition 2.1. a) Let us suppose that $m=m_{b}$ is a prime number and $m_{w}$ an even number. For each prime number $s$, such that

$$
4\left(t-t_{0}\right)+m \leq s \leq 8\left(t-t_{0}\right)+m,
$$

the system

$$
\begin{aligned}
s & =m+8 x+6 y+4 z, \\
t-t_{0} & =x+y+z,
\end{aligned}
$$

has solution.

b) Now let $m_{b}$ be even and $m=m_{w}$ prime. For each prime number $s$, such that

$$
4\left(t-t_{0}\right)+m \leq s \leq 8\left(t-t_{0}\right)+m,
$$

the system

$$
\begin{aligned}
s & =m+4 x+6 y+8 z, \\
t-t_{0} & =x+y+z
\end{aligned}
$$

has solution.

Proof. a) The number $s-m$ is an even number such that

$$
4\left(t-t_{0}\right) \leq s-m \leq 8\left(t-t_{0}\right)
$$

We may see that if $s-m \leq 6\left(t-t_{0}\right)$ then

$$
x=0, \quad y=\frac{s-m-4\left(t-t_{0}\right)}{2}, \quad z=\frac{6\left(t-t_{0}\right)-(s-m)}{2},
$$

is a solution of the system $(2.1)$. If $s-m>6\left(t-t_{0}\right)$ then

$$
x=\frac{s-m-6\left(t-t_{0}\right)}{2}, \quad y=\frac{8\left(t-t_{0}\right)-(s-m)}{2}, \quad z=0
$$

is a solution of the system.

b) For system (2.2) the solutions are the same ones interchanging the roles of $x$ and $z$.

In the next Section we apply this procedure to $n=12 t+r, r=$ $0,1, \ldots, 11$, taking $t_{0}=1$ or 2 as it is indicated in each class. 


\section{The groups $A_{n}$ with $13 \leq n \leq 167$}

This Section is devoted to prove the main result.

Theorem 3.1. The group $A_{n}$ is an $M^{*}$-group for all $n \geq 13$.

Proof. We begin with $n=13$ and $n=14$. It is known that $A_{13}$ is an $M^{*}$-group [8], but we give a direct proof by means of the singular diagram shown in Figure 3.

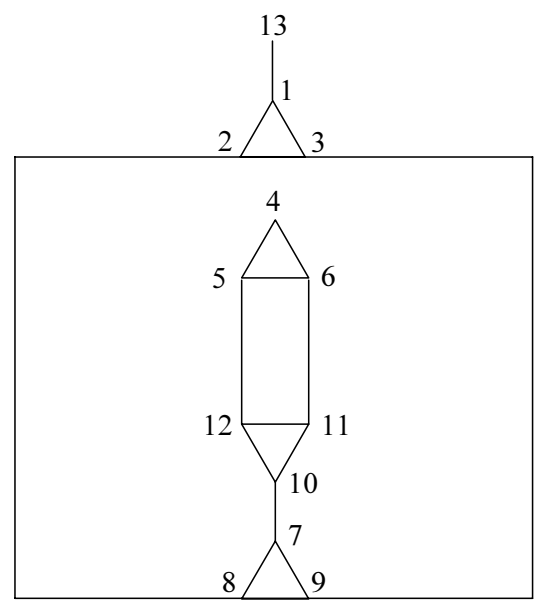

Figure 3.

According to the description in the previous Section, we have the following permutations:

$$
\begin{aligned}
X & =(1,2,3)(4,5,6)(7,8,9)(10,11,12)(13) \\
A & =(1,13)(2,8)(3,9)(4)(5,12)(6,11)(7,10) \\
B & =(1)(2,3)(4)(5,6)(7)(8,9)(10)(11,12)(13) .
\end{aligned}
$$

We are going to obtain the permutation $X A$ with the diagram. Recalling that $X$ runs in an anticlockwise order the triangles and $A$ runs the edges, we have that $X A$ is the composition of three cycles

$$
X A=(1,8,3,13)(2,9,10,6,4,12,7)(5,11) .
$$

We may see that there is a cycle of prime length 7 , this number does not divide the lengths of the other cycles and finally this cycle includes, for instance, 10 which is a fixed point of $B$. From Corollary B these permutations $X, A, B$, generate $A_{13}$ and it is an $M^{*}$-group.

We now prove that $A_{14}$ is also an $M^{*}$-group. Let us consider the Figure 4 and we proceed as in former group. 


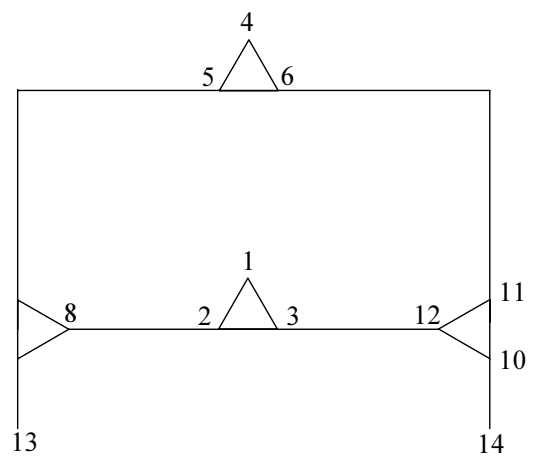

Figure 4.

The permutation $X A$ is $(1,8,5,11,3)(2,12,14,10,6,4,9,13,7)$. The 5 cycle contains the point 1 which is a fixed point of $B$. From Corollary B, $A_{14}$ is an $M^{*}$-group.

We shall come back to these two groups in Section 5 where we obtain all their possible sets of generators as $M^{*}$-groups.

From now on consider $n \geq 15, n=12 t+r, r=0,1, \ldots, 11, t_{0} \leq t \leq 13$. We run $r$ from 0 to 11 . In the first three cases $t_{0}=2$, and for the remaining cases $t_{0}=1$. We study the case $n=12 t$ in full detail to show the procedure. The remaining cases are similar and we give also the diagram and the cycle structure of $X A$ for $t_{0}$.

Case 12t. Let us observe Figure 5

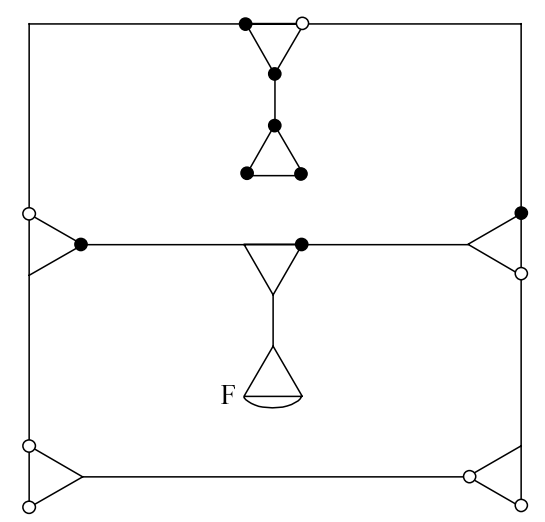

Figure 5.

We may see that the white cycle has length $m_{w}=7$, the black cycle has length $m_{b}=8$. The vertex $F$ is a fixed point of $X A$. The remaining vertices form a cycle of length 8 . This cycle will not change when we add blocks.

We write the cycle structure of $X A$ as $[7,8,8,1]$ and we denote as $m$ the prime length, in this case 7 . 
Now we see the general case for an arbitrary $t>t_{0}$. Remind that $t_{0}=2$. Let $s$ be a prime number between $7+4(t-2)$ and $7+8(t-2)$. It exists a cycle of $X A$ with length $s$ if and only if the following system has solution:

$$
\begin{aligned}
s & =7+4 x+6 y+8 z \\
t-2 & =x+y+z .
\end{aligned}
$$

This system has always solution by Proposition 2.1. We have that the structure of $X A$ for an arbitrary $t$ and each prime $s$ will be $[s, 12 t-$ $(s+9), 8,1]$. In order to see that the conditions in Corollary B are satisfied we must check that $s$ does not divide the length of another cycle. The only possibility would be $s$ dividing $|\mathcal{B}|$, but it is not possible because

$$
|\mathcal{B}|=8+8 x+6 y+4 z<2 s=14+8 x+12 y+16 z .
$$

Furthermore the cycle $\mathcal{W}$ always contains the two lowest points in the diagram which are paired by the generator $B$. Thus the conditions in the Corollary B are satisfied for each $t$ and each $s$ as above. In particular $A_{12 t}$ is an $M^{*}$-group for each $t \geq 2$.

For the remaining cases $r=1,2, \ldots, 11$, we give the diagram for $t_{0}$, the corresponding prime $m$ and the cycle structure of $X A$. We shall not mark the points of the cycles $\mathcal{B}$ and $\mathcal{W}$. Instead of we shall mark the points fixed of $B$ or the points in a cycle of $B$ which appear in the cycle of length prime in order to assure that the conditions in Corollary B are satisfied.

Case $12 \mathrm{t}+1$

We have $t_{0}=2, m=|\mathcal{B}|=11,|\mathcal{W}|=4$, and there is another cycle of length 10 . The cycle structure is $[11,4,10]$.

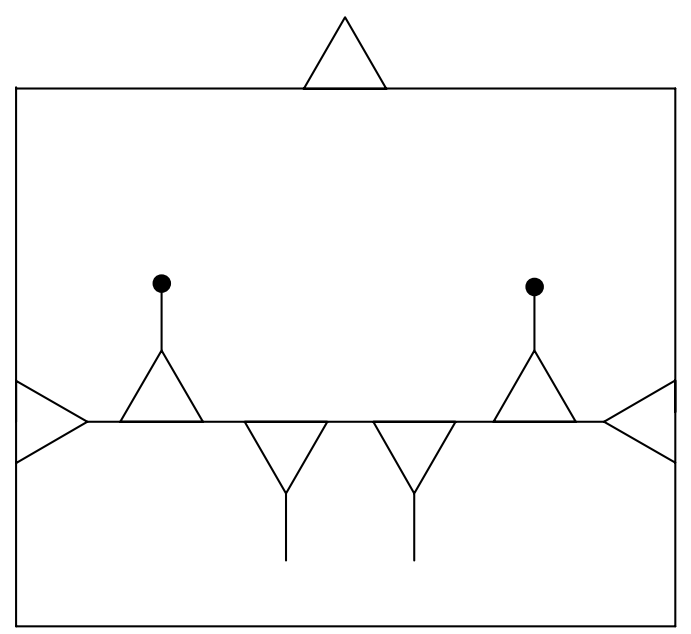

Figure 6. 
Case $12 \mathrm{t}+\mathbf{2}$

Now $t_{0}=2, m=|\mathcal{B}|=13,|\mathcal{W}|=6$, another cycle of length 6 and a fixed point of $X A$. The cycle structure is $[13,6,6,1]$.

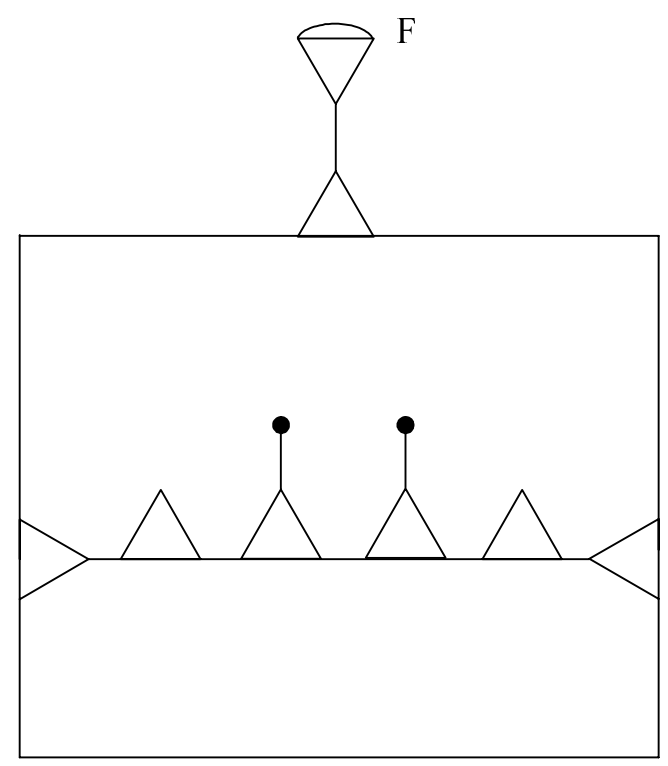

Figure 7.

From now on $t_{0}=1$.

Case $12 \mathrm{t}+3$

We see that $m=|\mathcal{B}|=7,|\mathcal{W}|=4$, and there is another cycle of length 4 . The cycle structure of $X A$ is $[7,4,4]$.

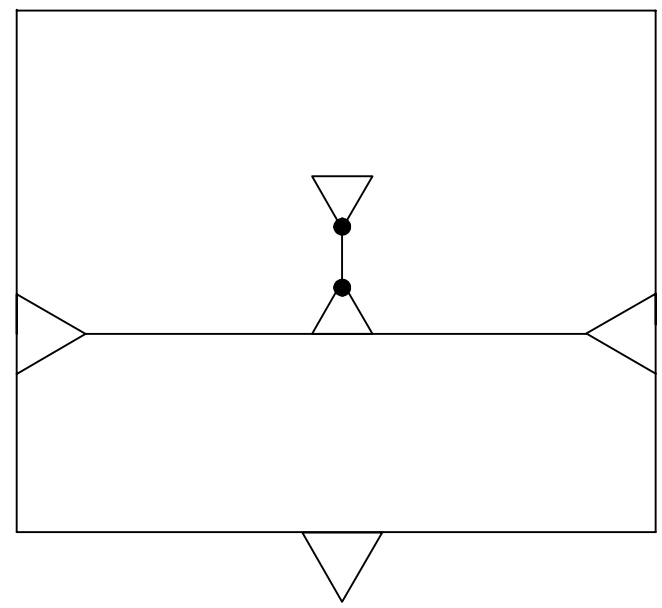

Figure 8. 
Case $12 \mathrm{t}+4$

In this case $m=|\mathcal{B}|=7,|\mathcal{W}|=2$, and we have two more cycles of length 3 and 4 . The cycle structure of $X A$ is $[7,2,3,4]$.

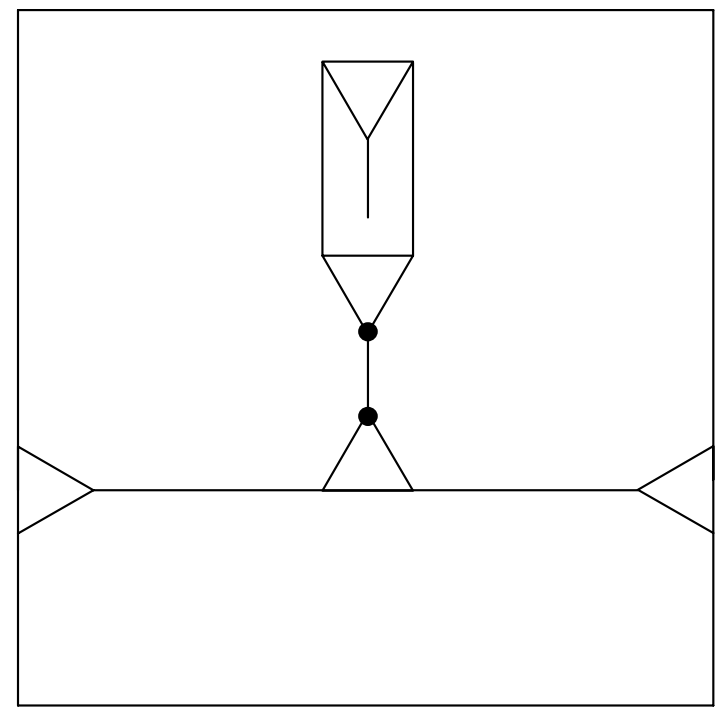

Figure 9.

Case $12 \mathrm{t}+5$

Here $m=|\mathcal{B}|=5,|\mathcal{W}|=6$ and there is another cycle of length 6 . The cycle structure of $X A$ is $[5,6,6]$.

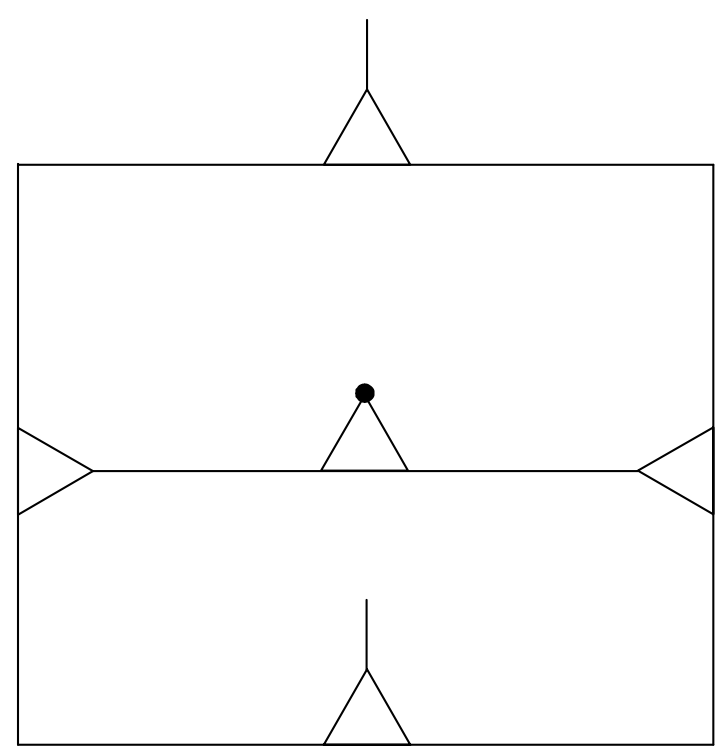

Figure 10. 
Case $12 \mathrm{t}+6$

In this case $m=|\mathcal{B}|=7,|\mathcal{W}|=2$, and there is another cycle of length 8 . There is also a fixed point $F$ of $X A$. The cycle structure of $X A$ is $[7,2,8,1]$.

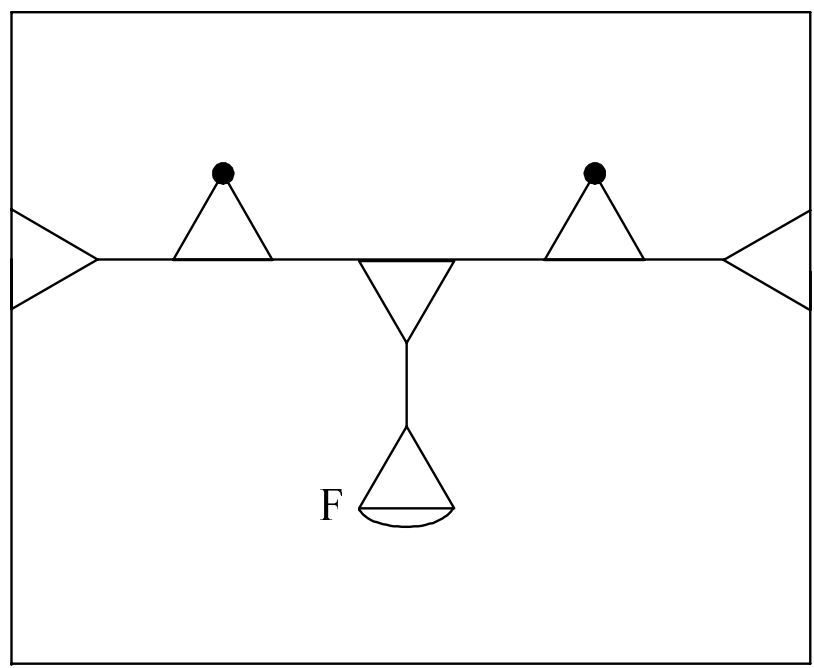

Figure 11.

Case $12 \mathrm{t}+7$

Now $m=|\mathcal{B}|=7,|\mathcal{W}|=8$, and we have another cycle of length 4 . The cycle structure of $X A$ is $[7,8,4]$.

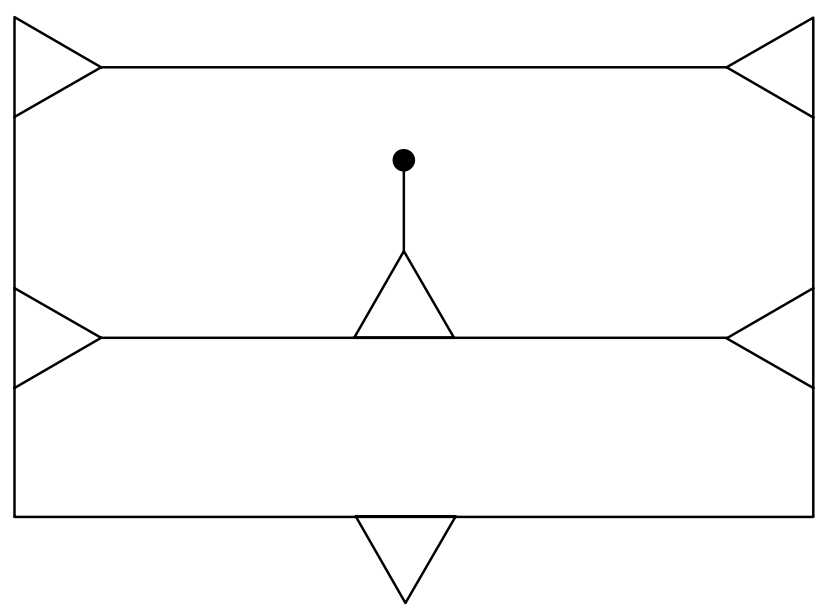

Figure 12 . 
Case $12 \mathrm{t}+8$

We have in this case $|\mathcal{B}|=4, m=|\mathcal{W}|=5$, and two cycles of lengths 8 and 3. The cycle structure of $X A$ is $[5,4,8,3]$.

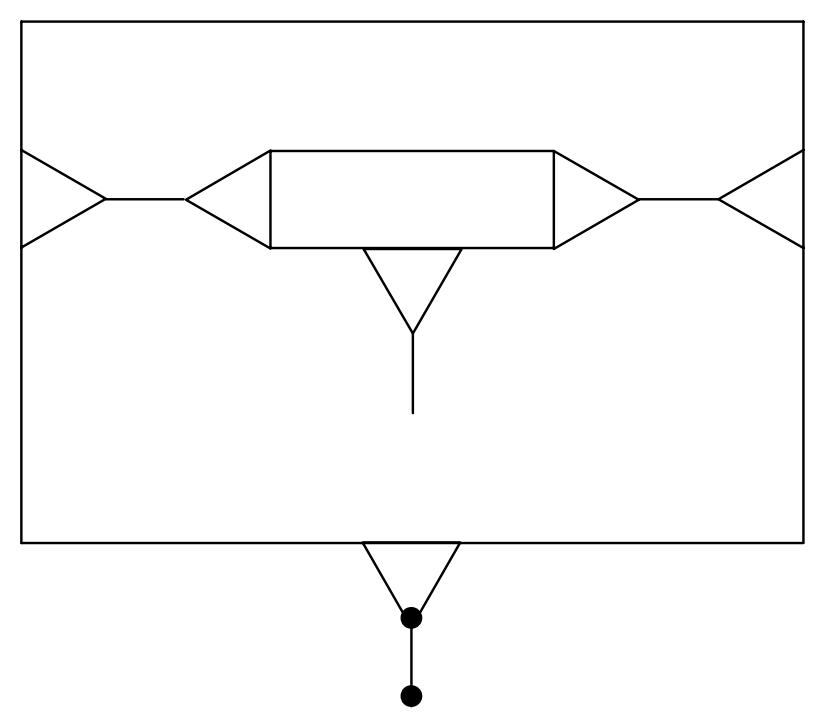

Figure 13.

Case $12 \mathrm{t}+9$

Now $m=|\mathcal{B}|=11,|\mathcal{W}|=4$, and there is another cycle of length 6 . The cycle structure of $X A$ is $[11,4,6]$.

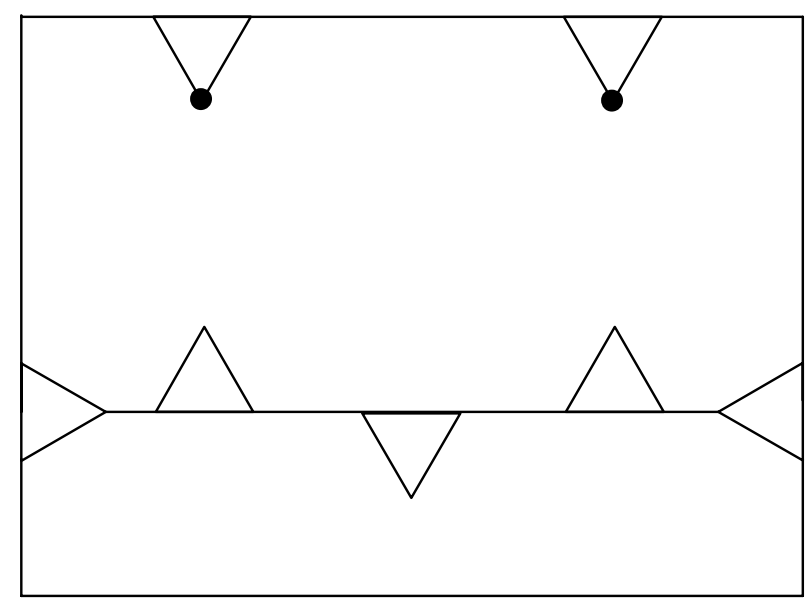

Figure 14 . 
Case $12 \mathrm{t}+10$

Here we have $m=|\mathcal{B}|=7,|\mathcal{W}|=8$, and two cycles of lengths 5 and 2 . The cycle structure of $X A$ is $[7,8,5,2]$.

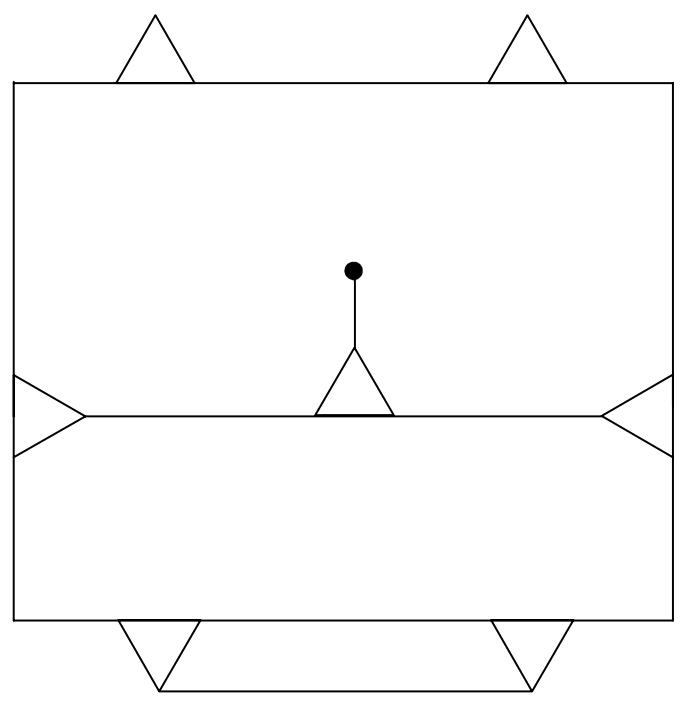

Figure 15.

Case $12 \mathrm{t}+11$

Finally, $|\mathcal{B}|=8, m=|\mathcal{W}|=11$, and there is another cycle of length 4 . The cycle structure of $X A$ is $[11,8,4]$.

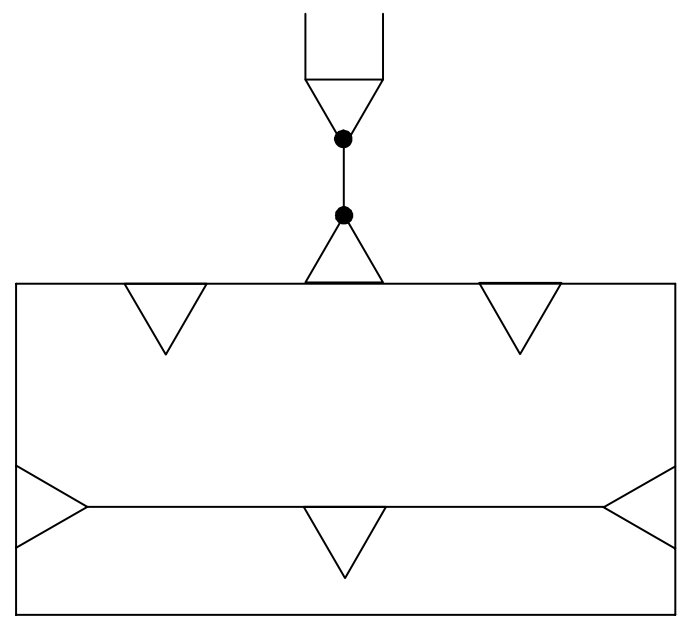

Figure 16.

Observe that in all twelve cases $2 m$ is greater than the length of the other relevant cycle, and so the prime length does not divide the other lengths. So the proof is finished and all $A_{n}, n \geq 13$, are $M^{*}$-groups. 
Remark 2. In [3, Section 5] Conder states that for $n>167, A_{n}$ is an $M^{*}$-group with order of $X A$ equal to 7 and that the same happens for some values of $n$. In [4, Section 4] he proves that for any $k>6$ there exists $N_{k}>0$ such that for every $n>N_{k}, A_{n}$ is an $M^{*}$-group with order of $X A$ equal to $k$. So the general question remained open for $n<168$.

Example. As an example of the procedure take, for instance, $n=130$. We have $r=10, t=10, t_{0}=1, m=|\mathcal{B}|=7,|\mathcal{W}|=8$, and cycle structure $[7,8,5,2]$, see Figure 15 .

The primes $s$ between $4\left(t-t_{0}\right)+m$ and $8\left(t-t_{0}\right)+m$ are in our case: $s=$ $43,47,53,59,61,67,71,73,79$. We summarize in Table 1 the cycle structure of $X A$ for each $s$ as well as the corresponding values of $x, y, z$, obtained from Proposition 2.1.

\begin{tabular}{|ccccc|}
\hline$s$ & & $x$ & $y$ & $z$ \\
\hline 43 & {$[43,80,5,2]$} & 9 & 0 & 0 \\
47 & {$[47,76,5,2]$} & 7 & 2 & 0 \\
53 & {$[53,70,5,2]$} & 4 & 5 & 0 \\
59 & {$[59,64,5,2]$} & 1 & 8 & 0 \\
61 & {$[61,62,5,2]$} & 0 & 9 & 0 \\
67 & {$[67,56,5,2]$} & 0 & 6 & 3 \\
71 & {$[71,52,5,2]$} & 0 & 4 & 5 \\
73 & {$[73,50,5,2]$} & 0 & 3 & 6 \\
79 & {$[79,44,5,2]$} & 0 & 0 & 9 \\
\hline
\end{tabular}

Table 1

We shall return to this example in Section 5.

\section{Groups $A_{n}$ with $n<13$}

In this Section we deal with the groups $A_{n}$ for $n<13$. It is already known that $A_{5}$ and $A_{10}$ are $M^{*}$-groups [8], and we shall come back to them in Section 5. For the other values of $n$ we prove that they are not $M^{*}$-groups.

As we saw in Section 1, to prove that a given group is not an $M^{*}$-group, we need to check that there exists no triple of generators $X, A, B$, satisfying that $X$ has order 3 , and $A, B, X B$ and $A B$ have order 2. Proposition $\mathrm{C}$ gives us the conditions that $B$ must fulfill.

Once obtained a suitable triple $(X, A, B)$, we recall that if the order of $X A$ is $p$ and the order of $X A B$ is $q$, the group $<X, A, B>$ is a quotient of $G^{3, p, q}$. For low values of $p$ and $q$ the groups $G^{3, p, q}$ are finite and of low order smaller than the relevant $A_{n}$. The only groups $G^{3, p, q}$ which appear in this 
Section and must be checked in another way are $G^{3,9,12}, G^{3,10,10}$ and $G^{3,10,12}$. In these cases we obtain via MAPLE the order of the group generated by the corresponding triple.

We are now ready to establish the following

Theorem 4.1. Let $n<13$. Then $A_{n}$ is an $M^{*}$-group if and only if $n=5$ or 10 .

Proof. The groups $A_{5}$ and $A_{10}$ were proved to be $M^{*}$-groups in [8]. We must check the other values of $n$.

- $A_{6}$ and $A_{7}$.

Their elements have order at most 7 , and the groups $G^{3, p, q}$ with both $p, q \leq 7$, have order at most 108 [6]. So $A_{6}$ and $A_{7}$ are not $M^{*}$-groups.

- $A_{8}$.

Its elements have order at most 7 or else 15 . In addition to the former paragraph, $G^{3,7,15}$ has order 12180 . So the unique possibility is that $A_{8}$ be a quotient of $G^{3,15,15}$. Then the order of $X A$ must be 15 . It is easy to check that this only holds if

$$
\begin{aligned}
X & =(1,2,3)(5,7,8) \\
A & =(4,5)(6,7)
\end{aligned}
$$

but then there exists no $B$ such that $B, X B$ and $A B$ have order 2 . So $A_{8}$ is not an $M^{*}$-group.

- $A_{9}$.

We look for the possible sets of generators $X, A, B$. Let first

$$
X=(1,2,3)(4,5,6)(7,8,9) \text {; }
$$

then by Proposition C

$$
B=(1)(2,3)(4,7)(5,9)(6,8),
$$

and there are four possible permutations $A$ :

$$
\begin{aligned}
& A 1=(1)(2,4)(3,7)(5,9)(6,8), \\
& A 2=(1)(2,4)(3,7)(5,6)(9,8), \\
& A 3=(1)(2,4)(3,7)(5,8)(6,9) \\
& A 4=(1)(2,4)(3,7)(5)(9)(6)(8) .
\end{aligned}
$$


Since $A 3$ is obtained from $A 2 B$, and $A 4$ from $A 1 B$, in both cases interchanging the cycles $(4,5,6)$ and $(7,8,9)$, we need only to consider $A 1$ and $A 2$. Firstly $A 2$ : the element $X A 2$ has order 7 and $X A 2 B$ has order 9 , but $G^{3,7,9}$ has order 504 and $A 2$ is discarded. Finally $A 1$ : the element $X A 1$ has order 12 and $X A 1 B$ has order 9 . Since $G^{3,9,12}$ is not known to be finite, we check via MAPLE the order of the group generated by $X, A 1$ and $B$, which is 648 .

Now let

$$
X=(1)(2)(3)(4,5,6)(7,8,9) .
$$

Then there are two possibilities for $B$, namely

$$
\begin{aligned}
& (1)(2,3)(4,7)(5,9)(6,8), \\
& (1)(2)(3)(4)(7)(5,6)(8,9),
\end{aligned}
$$

but in both cases there exists no $A$ such that the generated subgroup be transitive. The same happens finally if

$$
\begin{aligned}
X & =(1)(2)(3)(4)(5)(6)(7,8,9), \\
B & =(1,2)(3,4)(5,6)(7)(8,9) .
\end{aligned}
$$

So we have obtained that $A_{9}$ is not an $M^{*}$-group.

- $A_{11}$.

Let

$$
X=(1,2,3)(4,5,6)(7,8,9)(10)(11) .
$$

Then $B$ must be

$$
(10,11)(1)(4)(7)(2,3)(5,6)(8,9)
$$

or

$$
(10)(11)(1)(2,3)(4,7)(5,9)(6,8) \text {. }
$$

In both cases there exists no suitable $A$ such that $<X, A, B>$ be transitive. The same happens for

$$
X=(1,2,3)(4,5,6)(7)(8)(9)(10)(11)
$$

and $B$ one of

$$
\begin{aligned}
& (7)(8)(9)(10,11)(1,4)(2,6)(3,5), \\
& (7)(1)(4)(2,3)(5,6)(8,9)(10,11),
\end{aligned}
$$

or finally

$$
(1)(4)(7)(8)(9)(10)(11)(2,3)(5,6) \text {. }
$$

So $A_{11}$ is not an $M^{*}$-group. 
- $A_{12}$

This is the most involved case. Since the procedure to follow has already been explained we sketch here the results.

a) Consider first

$$
X=(1,2,3)(4,5,6)(7,8,9)(10,11,12) \text {. }
$$

Then $B$ can be

$$
(1,4)(2,6)(3,5)(7,10)(8,12)(9,11)
$$

or

$$
(1)(4)(7)(10)(2,3)(5,6)(8,9)(11,12) \text {. }
$$

The first possible $B$ provides 24 possible permutations $A$. Once considered the orders of $X A$ and $X A B$ only a single triple must be checked, namely the given by

$$
A=(1)(4)(2)(6)(3,7)(5,10)(8,12)(9,11),
$$

but the group $<X, A, B>$ has order 120 .

In the second value for $B$ we obtain 17 possible permutations $A$ and only four cases need to be checked:

$$
\begin{aligned}
& A 1=(1,4)(7,10)(2,8)(3,9)(5,12)(6,11), \\
& A 2=(1,4)(7,10)(2,9)(3,8)(5,11)(6,12), \\
& A 3=(1)(4)(7)(10)(2,8)(3,9)(5,12)(6,11), \\
& A 4=(1)(4)(7)(10)(2,9)(3,8)(5,11)(6,12) .
\end{aligned}
$$

The orders of the generated groups are respectively 120,120, 576 and 576 .

b) The second possible $X$ is

$$
(1)(2)(3)(4,5,6)(7,8,9)(10,11,12) \text {. }
$$

Then

$$
B=(1)(4)(7)(10)(2,3)(5,6)(8,9)(11,12),
$$

and $A$ must be

$$
(1,4)(7,10)(2,8)(3,9)(5,11)(6,12)
$$

or

$$
(1,4)(7,10)(2,8)(3,9)(5,12)(6,11) \text {. }
$$

In both cases the group $\langle X, A, B\rangle$ is a quotient of $G^{3,8,10}$, which has order 4320 . 
c) Finally if

$$
X=(1)(2)(3)(4)(5)(6)(7,8,9)(10,11,12),
$$

then

$$
B=(1,2)(3,4)(5,6)(7,10)(8,12)(9,11)
$$

and

$$
A=(1,7)(2,10)(3,8)(4,12)(5,9)(6,11)
$$

But $X A$ has order 6 , and the group $G^{3,6, q}$ has order at most $3 q^{2}$, [6]. Hence $A_{12}$ is not an $M^{*}$-group.

We have then finished the proof and $A_{5}$ and $A_{10}$ are the only $M^{*}$-groups for $n<13$.

\section{The surfaces with maximal symmetry}

A surface which has an $M^{*}$-group $G$ as automorphism group is said to have maximal symmetry [11]. Of course the algebraic genus $\mathfrak{p}$ of the surface is determined by the order of the group, because this order is $12(\mathfrak{p}-1)$. We are going to see that the surface is topologically determined by the information we get from the $M^{*}$-group $A_{n}$ with partial presentation:

$$
<X, A, B \mid X^{3}=A^{2}=B^{2}=(X B)^{2}=(A B)^{2}=(X A)^{p}=(X A B)^{q}=1, \ldots>
$$

The algebraic genus $\mathfrak{p}$ of the surface is related with its topological type, by means of the formula

$$
\mathfrak{p}=\eta g+k-1,
$$

where $g$ is the topological genus, $k$ is the number of boundary components, and $\eta=2$ if the surface is orientable or $\eta=1$ if non-orientable.

First of all consider the orientability. If $G$ acts on an orientable surface $G$ has a subgroup of index 2. Hence the simple groups act only on non-orientable surfaces. In our case the surface is always non-orientable, and so $\mathfrak{p}=g+k-1$.

It suffices now to obtain $k$ for having also $g$. By [11] and [7], $k=o(G) / 2 p$ and, by interchanging $A$ and $A B$, there is another surface with $k=o(G) / 2 q$.

Thus to know the topological types of surfaces with maximal symmetry having $A_{n}$ as automorphism group is equivalent to have all partial presentations of $A_{n}$ as a quotient of $G^{3, p, q}$. Explicitly

$$
\frac{n !}{2}=12(\mathfrak{p}-1)
$$


and so

$$
\frac{n !}{24}+1=\mathfrak{p}=g+k-1,
$$

where $k=\frac{n !}{2 \cdot 2 p}$ and $k=\frac{n !}{2 \cdot 2 q}$.

This knowledge of each $A_{n}$ as a quotient of $G^{3, p, q}$ requires an individual study group by group, and it becomes much harder as $n$ grows. We make it here explicitly for the four first values of $n$, precisely 5, 10, 13 and 14 .

- $A_{5}$.

Necessarily

$$
\begin{aligned}
X & =(1,2,3)(4)(5) \\
B & =(1)(2,3)(4,5), \\
A & =(1)(2,4)(3,5) .
\end{aligned}
$$

Then $X A=(1,4,2,5,3)$ and $X A B=(1,5,2,4,3)$. The group generated by $X, A, B$ is a quotient of $G^{3,5,5}$. Besides it includes elements of orders 2,3 and 5 and so its order is a multiple of 30 . So $<X, A, B\rangle=A_{5}$. In fact, Coxeter pointed out in [6] that $A_{5} \approx G^{3,5,5}$. This is the unique way to obtain $A_{5}$ as an $M^{*}$-group, and the surface is non-orientable and has algebraic genus 6 , topological genus 1 , and 6 boundary components.

- $A_{10}$.

Let first

$$
X=(1,2,3)(4,5,6)(7,8,9)(10)
$$

Then

$$
B=(10)(1)(2,3)(4,7)(5,9)(6,8),
$$

and there are two possible $A$. If

$$
A=(10,1)(4)(7)(2,5)(3,9)(6,8),
$$

$X A$ has order 5: we discard it since $G^{3,5, q}$ has order at most 120 . If

$$
A=(10,1)(4)(7)(2,9)(3,5)(6,8),
$$

then

$$
\begin{aligned}
X A & =(1,9,7,6,4,3,10)(2,5,8), \\
X A B & =(1,5,6,7,8,3,10)(2,9,4),
\end{aligned}
$$

both of order 21. Besides one applies Corollary B to the 7-cycle of $X A$, and hence $\langle X, A, B\rangle=A_{10}$ is a quotient of $G^{3,21,21}$. The surface has algebraic 
genus $\frac{10 !}{24}+1$ with $\frac{10 !}{84}$ boundary components. This is the unique action of $A_{10}$ as an $M^{*}$-group because

$$
X=(1,2,3)(4,5,6)(7)(8)(9)(10)
$$

gives

$$
B=(1)(4)(2,3)(5,6)(7,8)(9,10),
$$

or

$$
(7)(8)(1,4)(2,6)(3,5)(9,10),
$$

but in none case there exists $A$ such that $<X, A, B>$ be transitive.

- $A_{13}$.

This is the first case in which several actions exist. Let first

$$
X=(1,2,3)(4,5,6)(7,8,9)(10,11,12)(13) .
$$

Then $B$ is necessarily

$$
(13)(1)(4)(7)(10)(2,3)(5,6)(8,9)(11,12) .
$$

We now have four possibilities for $A$, namely

$$
\begin{aligned}
& A 1=(13,1)(4)(7,10)(2,8)(3,9)(5,11)(6,12), \\
& A 2=(13,1)(4)(7,10)(2,8)(3,9)(5,12)(6,11), \\
& A 3=(13,1)(4)(7,10)(2,9)(3,8)(5,11)(6,12), \\
& A 4=(13,1)(4)(7,10)(2,9)(3,8)(5,12)(6,11),
\end{aligned}
$$

and since $A 3$ and $A 4$ are obtained from $A 1$ and $A 2$ by replacing $A$ by $A B$ we consider only the two former.

First, take $A 2$ :

$$
\begin{aligned}
X A 2 & =(1,8,3,13)(2,9,10,6,4,12,7)(5,11), \\
X A 2 B & =(1,9,10,5,12,7,3,13)(2,8)(4,11,6),
\end{aligned}
$$

and applying Corollary B to the 7 -cycle of $X A 2$ we have that $\langle X, A 2, B\rangle=$ $A_{13}$ is a quotient of $G^{3,24,28}$. Note that these generators correspond to the diagram in Figure 3, seen in Section 3.

Now take $A 1$. The order of $X A 1$ is 12 and the order of $X A 1 B$ is 18 but these permutations have no cycle of prime length to apply Corollary B. However the element $B(A 1 X B)^{2}$ has order 13 . The only maximal subgroups of $A_{13}$ having elements of order 13 are $P S L(3,3)$ and $13: 6$, but none of them has elements of order 12. So also $\langle X, A 1, B\rangle=A_{13}$ and it is a quotient of $G^{3,12,18}$. Let us observe that Corollary B gives sufficient but not necessary conditions. 
Now consider

$$
X=(1,2,3)(4,5,6)(7,8,9)(10)(11)(12)(13) .
$$

Then

$$
B=(10,11)(12,13)(1)(2,3)(4,7)(5,9)(6,8) .
$$

We have again four possibilities for $A$ :

$$
\begin{aligned}
& A 1=(1)(10,4)(11,7)(12,5)(13,9)(2,6)(3,8), \\
& A 2=(1)(10,4)(11,7)(12,5)(13,9)(2,8)(3,6), \\
& A 3=(1)(10,4)(11,7)(12,6)(13,8)(2,5)(3,9), \\
& A 4=(1)(10,4)(11,7)(12,6)(13,8)(2,9)(3,5),
\end{aligned}
$$

and $A 2$ and $A 4$ are obtained from $A 1$ and $A 3$ by substituting $A$ by $A B$, and $(10,11)$ by $(12,13)$. One obtains the orders of $X A 1, X A 1 B, X A 3$ and $X A 3 B$ all equal to 13 . Now $B(A 1 X B)^{2}$ and $B(A 3 X B)^{2}$ have order 18 . Neither $P S L(3,3)$ nor $13: 6$ have elements of order 18 . Hence both $\langle X, A 1, B\rangle$ and $<X, A 3, B>$ are $A_{13}$, and it is a quotient of $G^{3,13,13}$.

Finally if $X$ were

$$
(1,2,3)(4,5,6)(7)(8)(9)(10)(11)(12)(13),
$$

$B$ could be

$$
(1,4)(2,6)(3,5)(7,8)(9,10)(11,12)(13)
$$

or

$$
(1)(2,3)(4)(5,6)(7)(8)(9)(10,11)(12,13),
$$

but in no case there exists a suitable $A$ with $\langle X, A, B\rangle$ transitive.

Hence $A_{13}$ admits $M^{*}$-group presentations as a quotient of $G^{3,24,28}, G^{3,12,18}$ and $G^{3,13,13}$ and it acts on surfaces of algebraic genus $\frac{13 !}{24}+1$, with $\frac{13 !}{96}, \frac{13 !}{112}, \frac{13 !}{48}$, $\frac{13 !}{72}$ and $\frac{13 !}{52}$ boundary components, respectively.

- $A_{14}$.

We may proceed as the previous group. Since much more cases appear we only give the generating triples.

Let first

$$
X=(1,2,3)(4,5,6)(7,8,9)(10,11,12)(13)(14) \text {, }
$$

then the permutation $B$ is either

$$
(13,14)(1)(4)(2,3)(5,6)(7,10)(8,12)(9,11),
$$

or

$$
(13)(14)(1)(4)(7)(10)(2,3)(5,6)(8,9)(11,12) \text {. }
$$


In the first case one gets twelve possible values for $A$, of which five provide generating triples as follows:

$$
\begin{aligned}
A 1 & =(13,2)(14,3)(1,4)(5,7)(6,10)(8,12)(9)(11) \\
A 2 & =(13,2)(14,3)(1,4)(5,7)(6,10)(8)(12)(9,11) \\
A 3 & =(13,7)(14,10)(1)(4)(2,8)(3,12)(5,9)(6,11) \\
A 4 & =(13,7)(14,10)(1)(4)(2,12)(3,8)(5,11)(6,9) \\
A 5 & =(13,7)(14,10)(1,4)(2)(3)(5,12)(6,8)(9,11)
\end{aligned}
$$

Let us study them by order:

$$
X A 1=(1,8,5,11,3)(2,12,14,10,6,4,9,13,7),
$$

and the 5 -cycle contains the point 1 fixed by $B$. So one applies Corollary B and since $X A 1 B$ has order 45 , the group $<X, A 1, B>=A_{14}$ is a quotient of $G^{3,45,45}$.

Now $X A 2=(1,13,2,14,3,4,7,8,11,12,6)(5,10,9)$ and the 11-cycle obviously satisfies Corollary B. $X A 2 B$ has order 33 and $\langle X, A 2, B\rangle=A_{14}$ is a quotient of $G^{3,33,33}$.

The element $X A 3=(1,8,5,11,3)(2,12,14,10,6,4,9,13,7)$. Again the 5 -cycle contains the point 1 , and $X A 3 B$ has order 45 : a new presentation as a quotient of $G^{3,45,45}$. These generators correspond to Figure 4 in Section 3.

We deal with $A 4$. Then $X A 4=(1,12,14,10,5,9,13,7,3)(2,8,6,4,11)$, and the 5-cycle contains 4 , a fixed point of $B$. Since $X A 4 B$ has order 45 , we have a third presentation of $A_{14}$ as a quotient of $G^{3,45,45}$.

Let now take $A 5$, then $X A 5=(1,2,3,4,12,14,10,9,13,7,6)(5,8,11)$. Obviously the 11-cycle satisfies Corollary B, and since $X A 5 B$ has order 13 , the group $<X, A 5, B\rangle=A_{14}$ is a quotient of $G^{3,13,33}$.

The other possible values of $X$ do not give generating triples and so we have obtained that $A_{14}$ is a quotient of $G^{3,45,45}, G^{3,33,33}, G^{3,13,33}$ and $G^{3,60,60}$ and it acts on surfaces of algebraic genus $\frac{14 !}{24}+1$, with $\frac{14 !}{52}, \frac{14 !}{132}, \frac{14 !}{180}$ and $\frac{14 !}{240}$ boundary components.

For bigger $n$, the procedure given in Section 3 provides a large number of values of $p$ and $q$. See the example on $A_{130}$ where the cycle structure of $X A$ is displayed in Table 1 and gives nine different values for $p$. However there are much more possible values, since the obtained ones have been got with just a single diagram as departure, and besides there are presentations not satisfying the sufficient conditions of Corollary $\mathrm{B}$ (see $A_{13}$ above). Hence for a given $n$ one must follow the procedure in this Section to obtain all corresponding surfaces. 


\section{The determination of the real genus}

A finite group $G$ may act as an automorphism group of different bordered Klein surfaces. The minimum algebraic genus of these surfaces is called the real genus of $G$ and it is denoted by $\rho(G)$. Obviously, $\rho(G) \geq \frac{o(G)}{12}+1$. The systematical study of the real genus was begun by C. L. May in [16]. Two kinds of problems appear. Firstly to obtain the groups with real genus $n$ for each natural number $n$. On the other hand, for each given family of finite groups, to calculate the real genus of each member in that family.

Up to now, the groups such that $0 \leq \rho(G) \leq 8$ have been calculated. Groups with real genus 0 are $C_{n}$ and $D_{n}$ (cyclic groups and dihedral groups, respectively). The groups with real genus 1 are $C_{2} \times C_{n}$ for $n \geq 4$ even, and $C_{2} \times D_{n}$ for $n$ even. If $\rho(G) \geq 2$, the number of groups for each genus is finite. There is no group with real genus 2 . The groups with real genus 3 are $S_{4}$ and $A_{4}$. For $\rho(G)=4,5$, see [15], [16] and [17] and a correction in [19]. For genus 6,7 and 8, see [12] and a couple of amendments in [10]. The real genus of several families of groups is also known. In particular, for simple groups, D. Singerman in [21] determined the groups $P S L(2, q)$ which are $M^{*}$-groups, and C. L. May obtained in [18] the real genus of the remaining groups $P S L(2, q)$. In that paper he gives a list of open problems, the first of which is to determine the real genus of the alternating groups $A_{n}$. He recalls the results of Conder for $n \geq 168$ and questions for the real genus of $A_{n}$ when $n<168$. In Theorems 3.1 and 4.1, we have proved that all simple groups $A_{n}$ are $M^{*}$-groups with the only exceptions of $n=6,7,8,9,11,12$. So excepting these values of $n$,

$$
\rho\left(A_{n}\right)=\frac{n !}{24}+1
$$

Since May obtained in [18] that $\rho\left(A_{6}\right)=61$, in this Section we obtain the real genus of the five missing groups.

The most useful tool for obtaining finite groups as automorphism groups of Klein surfaces is the theory of NEC groups. For a general work of this topic see [1, Chapter 4]. To be precise, if $G$ is an automorphism group of a Klein surface of algebraic genus

$$
\mathfrak{p} \leq \frac{o(G)}{24 / 5}+1
$$

then $G$ is an image of an NEC group $\Gamma$ whose signature $\sigma(\Gamma)$ is in the following table, in such a way that the kernel of epimorphism is a surface group [1, Lemma 4.1.1]: 


$$
\begin{array}{cc}
\sigma(\Gamma) & \mathfrak{p} \\
(0,+,[-],\{(2,2,2, n)\}) & \frac{o(G)}{4 n /(n-2)}+1 \\
(0,+,[-],\{(2,2,3,3)\}) & \frac{o(G)}{6}+1 \\
(0,+,[3],\{(2,2)\}) & \frac{o(G)}{6}+1 \\
(0,+,[2,3],\{(-)\}) & \frac{o(G)}{6}+1 \\
(0,+,[-],\{(2,2,3,4)\}) & \frac{o(G)}{24 / 5}+1
\end{array}
$$

So for each of the five alternating groups we study whether they are an image of these NEC groups following the same order. This question depends on the existence of the certain partial presentation of $A_{n}$. For, $G$ is such an image of $(0,+,[-],\{(2,2, m, n)\})$ if and only if it can be generated by three elements $a, b, c$ satisfying $a^{2}=b^{2}=c^{2}=(a b)^{m}=(a c)^{n}=1$ (see [1, Lemma 4.1.2]). Observe that $m=2, n=3$ gives $\mathfrak{p}=\frac{o(G)}{12}+1$. This is the reason why this presentation provides the $M^{*}$-groups.

In the same way $G$ is an image of $(0,+,[3],\{(2,2)\})$ or $(0,+,[2,3],\{(-)\})$ if and only if it is generated by two elements of orders 2 and 3 . We only arrive to this level for the group $A_{8}$, but this is not a $(2,3)$-group, [20].

Hence we shall always be concerned by the existence of a triple $a, b, c$ satisfying $a^{2}=b^{2}=c^{2}=(a b)^{m}=(a c)^{n}=1$. We call $G_{a b c}$ the group generated by the triple, and check whether $G_{a b c}$ is the relevant group $A_{n}$. For brevity, we call $[2,2, m, n]$ the group with signature $(0,+,[-],\{(2,2, m, n)\})$.

- $A_{7}$.

First of all we prove that $A_{7}$ is not an image of $[2,2,2,4]$. Let us suppose the contrary. Then it should have generators $a, b, c$, such that $a^{2}=b^{2}=c^{2}=$ $(a b)^{2}=(a c)^{4}=1$. Call $p=o(b c)$ and $q=o(a b c)$. Then $A_{7}$ is a quotient of $G^{4, p, q}$. Because the elements of $A_{7}$ have order at most 7 we deduce from [6] that $p$ and $q$ must be 6 or 7 . Furthermore the group $A_{7}$ does not contain any dihedral subgroup $D_{7}$. So $p=q=6$.

Let $b c=(1,2,3)(4,5)(6,7)$. Then $b$ must be $(1,2)(4,5)$ and $c=(1,3)(6,7)$. The element $a$ is then $(1,2)(3,6)$ or $(4,5)(3,6)$. In both cases the group generated by $a, b$ and $c$ is not transitive.

Now we see that $A_{7}$ is not an image of $[2,2,2,5]$. Let us suppose the contrary. Since the element $a c$ has order 5 it must be $(1,2,3,4,5)$. At least one of these points must be fixed by $a$. Let us take 1 as fixed point. Then $a=(2,5)(3,4)$ and so $c=(1,2)(3,5)$. Then $b$ applies 6 to 7 or one of them is a fixed point. In both cases the generated group is not transitive. 
We prove now that $A_{7}$ is an image of $[2,2,3,3]$. We define

$$
\begin{aligned}
a & =(1,2)(4,5), \\
b & =(1,3)(4,6), \\
c & =(1,6)(4,7) .
\end{aligned}
$$

We have $a, b$ and $c$ of order 2 , and $a b$ and $a c$ of order 3 . Besides $b c=$ $(1,3,6,7,4)$ and $a b c=(1,2,3,6,7,4,5)$. The generated group $G_{a b c}$ has elements with orders $2,3,5$ and 7 , and so its order is a multiple of 210 , but $A_{7}$ has no proper subgroup with such an order. We have proved that

$$
\rho\left(A_{7}\right)=\frac{o\left(A_{7}\right)}{6}+1=421 .
$$

- $A_{8}$.

We start proving that $A_{8}$ is not an image of $[2,2,2,4]$. If this were the case it would be generated by elements $a, b, c$, satisfying $a^{2}=b^{2}=c^{2}=$ $(a b)^{2}=(a c)^{4}=1$. First consider $a c$. We have two possibilities:

$$
\begin{aligned}
& a c=(1,2,3,4)(5,6), \\
& a c=(1,2,3,4)(5,6,7,8) .
\end{aligned}
$$

In the first case there are three possibilities for $a$ and hence for $c$. For each $a$ we calculate $b$. The procedure provides the following candidate triples:

$$
\begin{array}{llc}
\multicolumn{1}{c}{a} & \multicolumn{1}{c}{c} & \multicolumn{1}{c}{b} \\
(2,4)(5,6) & (1,2)(3,4) & (1,7)(2,5)(3,8)(4,6) \\
(1,3)(7,8) & (1,4)(2,3)(5,6)(7,8) & (1,7)(2,5)(3,8)(4,6) \\
(1,2)(3,4)(5,6)(7,8) & (1,3)(7,8) & (1,5)(2,6)(3,7)(4,8)
\end{array}
$$

In the first two sets the order of $b c$ or of $a b c$ is 4 , and so the group generated by these elements is a quotient of $G^{4,4, q}$ which has order lower than $o\left(A_{8}\right)$. We may discard these triples. In the last case this argument fails because $o(b c)=o(a b c)=6$, and $G^{4,6,6}$ is infinite. Instead of we obtain via MAPLE the order of $G_{a b c}$, and that order is 192 .

In the second case there are three (non equivalent) possibilities for $a$. Once determined $c$ we calculate $b$ and a lot of possible $b$ are obtained. If $o(b c)$ or $o(a b c)$ are lower than 6 the triple is discarded. Finally we have the remaining triples. Again we check via MAPLE the order of the generated groups. The result is displayed in the following Table:

\begin{tabular}{lllc}
\multicolumn{1}{c}{$a$} & \multicolumn{1}{c}{$c$} & \multicolumn{1}{c}{$b$} & $o\left(G_{a b c}\right)$ \\
$(2,4)(6,8)$ & $(1,2)(3,4)(5,6)(7,8)$ & $(1,5)(6,8)$ & 192 \\
$(2,4)(6,8)$ & $(1,2)(3,4)(5,6)(7,8)$ & $(1,5)(2,4)$ & 192 \\
$(1,2)(3,4)(5,6)(7,8)$ & $(1,3)(5,7)$ & $(1,5)(2,6)(3,8)(4,7)$ & 48 \\
$(1,2)(3,4)(5,6)(7,8)$ & $(1,3)(5,7)$ & $(1,6)(2,5)(3,7)(4,8)$ & 48 \\
$(1,5)(2,8)(3,7)(4,6)$ & $(1,6)(2,5)(3,8)(4,7)$ & $(3,7)(4,6)$ & 48
\end{tabular}


We may conclude that $A_{8}$ is not an image of $[2,2,2,4]$.

Consider now $[2,2,2,5]$. In this case $a c$ has order 5 . Let $a c=(1,2,3,4,5)$. If $a$ has not fixed points then it must have a cycle including just one fixed point of $a c$, for instance, the cycle is $(1,6)$. In such a case there does not exist $c$ of order 2. So that $a$ must have fixed points, exactly four points. At least one of them must appear in the 5 -cycle of $a c$. Let us take 1 . Then $a=(2,5)(3,4), c=(1,2)(3,5)$. Now $b$ applies the fixed points of $a$ among themselves. Since the points $6,7,8$ are fixed by $a$ and by $c$, and two of them are either fixed points or form a 2-cycle of $b$, the group $G_{a b c}$ is not transitive.

We now study the case $[2,2,2,6]$. Reasoning as above there appear nine possibilities for the triple $(a, b, c)$. The orders of the groups $G_{a b c}$ are checked via MAPLE and they are at most 576. Hence $A_{8}$ is not an image of $[2,2,2,6]$.

The following case to be considered is $[2,2,3,3]$. The two possibilities in this case are discarded in the same way.

The next case is $[2,2,3,4]$. Let

$$
\begin{aligned}
a & =(2,4)(7,8), \\
b & =(2,5)(6,7), \\
c & =(1,2)(3,4)(5,6)(7,8) .
\end{aligned}
$$

We have $a, b$ and $c$ of order $2, a b=(2,4,5)(6,7,8)$ of order 3 and $a c=$ $(1,2,4,3)(5,6)$ of order 4 . Besides the elements

$$
\begin{aligned}
a b c & =(1,2,3,4,6,8,5), \\
c(a b a c b)^{2} & =(1,5,6,4,7)(2,3,8),
\end{aligned}
$$

have order 7 and 15, respectively. There exists no proper subgroup in $A_{8}$ having elements of order 7 and 15 . Hence $a, b, c$ generate $A_{8}$.

The real genus of $A_{8}$ is then

$$
\rho\left(A_{8}\right)=\frac{8 !}{2} \frac{5}{24}+1=4201 .
$$

- $A_{9}$.

This group is an image of $[2,2,2,4]$. To prove it we define $a, b, c$ as

$$
\begin{aligned}
& a=(2,4)(5,6), \\
& b=(1,9)(2,5)(3,7)(4,6), \\
& c=(1,2)(3,4)(5,6)(7,8) .
\end{aligned}
$$

These elements satisfy $a b$ of order 2 and $a c$ of order 4 . Besides the element $b c=(1,9,2,6,3,8,7,4,5)$ has order 9 and $a(b c)^{2}=(1,2)(3,7,5,8,4)(6,9)$ 
has order 10. The only maximal subgroups of $A_{9}$ with order a multiple of 10 are $A_{8}, S_{7},\left(A_{6} \times 3\right): 2$ and $\left(A_{5} \times A_{4}\right): 2$. No one of them has elements of order 9 and so the generated group $G_{a b c}$ is $A_{9}$.

The real genus of $A_{9}$ is

$$
\rho\left(A_{9}\right)=\frac{9 !}{2 \cdot 8}+1=22681 .
$$

- $A_{11}$.

We must first check whether it is an image of $[2,2,2,4]$. By straightforward calculation we obtain that all suitable triples generate non transitive groups. So we now deal with $[2,2,2,5]$. We take

$$
\begin{aligned}
a & =(2,5)(3,4)(7,10)(8,9), \\
b & =(1,11)(3,9)(4,8)(7,10), \\
c & =(1,2)(3,5)(6,7)(8,10) .
\end{aligned}
$$

These elements satisfy $a b$ of order 2 and $a c$ of order 5 . Furthermore $a(b c)^{2}=$ $(1,2,9,10,4,5,11)(3,6,8)$ has order 21 and

$$
\text { abcacbc }=(1,2,10,5)(3,7,8,11,9)(4,6)
$$

has order 20. The group $A_{11}$ has no subgroup containing simultaneously elements of order 20 and 21 . Then the generated group $G_{a b c}$ is $A_{11}$.

The real genus of $A_{11}$ is

$$
\rho\left(A_{11}\right)=\frac{11 !}{2 \cdot \frac{20}{3}}+1=2993761 .
$$

- $A_{12}$.

First we check whether it is an image of $[2,2,2,4]$. There are 25 nonequivalent triples which are candidates to generate our group. In all cases MAPLE gives the order of the generated group to be very smaller than the order of $A_{12}$, so $A_{12}$ is not an image of $[2,2,2,4]$.

We now see that it is an image of $[2,2,2,5]$. Let

$$
\begin{aligned}
a & =(2,5)(3,4)(7,10)(8,9), \\
b & =(1,11)(2,8)(3,4)(5,9)(6,12)(7,10), \\
c & =(1,2)(3,5)(6,7)(8,10) .
\end{aligned}
$$

These elements satisfy $a b$ of order 2 and $a c$ of order 5 . Besides the elements $b c$ and $a b c a c b c$ are respectively $(1,11,2,10,6,12,7,8)(3,4,5,9)$ and $(1,2,6,7,8,5,12)(3,11,4,9,10)$ of order 8 and 35 respectively. The unique maximal subgroup of $A_{12}$ having elements of order 35 is $\left(A_{7} \times A_{5}\right): 2$, but it has not elements of order 8 . Hence the generated group $G_{a b c}$ is $A_{12}$. 
The real genus of $A_{12}$ is

$$
\rho\left(A_{12}\right)=\frac{12 !}{2 \cdot \frac{20}{3}}+1=35925121 .
$$

We summarize these results in the following

Theorem 6.1. Let $n \geq 5$. The alternating group $A_{n}$ has real genus $\rho\left(A_{n}\right)$ $=\frac{n !}{24}+1$, with the following exceptions:

$\begin{array}{lr}A_{6} & 61 \\ A_{7} & 421 \\ A_{8} & 4201 \\ A_{9} & 22681 \\ A_{11} & 2993761 \\ A_{12} & 35925121\end{array}$

\section{References}

[1] Bujalance, E., Etayo, J. J., Gamboa, J. M. and Gromadzki, G.: Automorphism groups of compact bordered Klein surfaces. A combinatorial approach. Lecture Notes in Mathematics 1439. Springer-Verlag, Berlin, 1990.

[2] Conder, M. D. E.: Minimal generating pairs for permutation groups. Ph. D. Thesis, University of Oxford, 1980.

[3] Conder, M. D. E.: Generators for alternating and symmetric groups. J. London Math. Soc. (2) 22 (1980), 75-86.

[4] Conder, M. D. E.: More on generators for alternating and symmetric groups. Quart. J. Math. Oxford Ser. (2) 32 (1981), 137-163.

[5] Conder, M. D. E.: The symmetric genus of alternating and symmetric groups. J. Combin. Theory Ser. B 39 (1985), 179-186.

[6] Coxeter, H. M. S.: The abstract groups $G^{m, n, p}$. Trans. Amer. Math. Soc. 45 (1939), 73-150.

[7] Etayo, J. J.: Klein surfaces with maximal symmetry and their groups of automorphisms. Math. Ann. 268 (1984), no. 4, 533-538.

[8] Etayo, J. J.: Una nota sobre $M^{*}$-grupos simétricos y alternados. In Actas XII Jornadas Luso-Españolas de Matemáticas (Braga 1987) II, 62-67, 1989.

[9] Etayo, J. J. And Martínez, E.: Alternating groups, Hurwitz groups and $H^{*}$-groups. J. Algebra 283 (2005), 327-349.

[10] Etayo, J. J. and Martínez, E.: The real genus of cyclic by dihedral and dihedral by dihedral groups. J. Algebra 296 (2006), 145-156.

[11] Greenleaf, N. And May, C. L.: Bordered Klein surfaces with maximal symmetry. Trans. Amer. Math. Soc. 274 (1982), 265-283. 
[12] Gromadzki, G. And Mockiewicz, B.: The groups of real genus 6, 7 and 8. Houston J. Math. 28 (2002), 691-699.

[13] MAY, C. L.: Large automorphism groups of compact Klein surfaces with boundary. I. Glasgow Math. J. 18 (1997), 1-10.

[14] MaY, C. L.: The species of bordered Klein surfaces with maximal symmetry of low genus. Pacific J. Math. 111 (1984), 371-394.

[15] May, C. L.: The groups of real genus 4. Michigan Math. J. 39 (1992), 219-228.

[16] May, C. L.: Finite groups acting on bordered surfaces and the real genus of a group. Rocky Mountain J. Math. 23 (1993), 707-724.

[17] MaY, C. L.: Groups of small real genus. Houston J. Math. 20 (1994), 393-408.

[18] May, C. L.: Real genus actions of finite simple groups. Rocky Mountain J. Math. 31 (2001), 539-551.

[19] May, C. L.: The real genus of 2-groups. J. Algebra Appl. 6 (2007), no. $1,103-118$.

[20] Miller, G. A.: On the groups generated by two operators. Bull. Amer. Math. Soc. 7 (1901), 424-426.

[21] Singerman, D.: $\operatorname{PSL}(2, q)$ as an image of the extended modular group with applications to group actions on surfaces. Proc. Edinburgh Math. Soc. (2) 30 (1987), 143-151.

[22] Wielandt, H.: Finite permutations groups. Academic Press, New YorkLondon, 1964.

Recibido: 21 de marzo de 2007.

Revisado: 14 de diciembre de 2007.

José Javier Etayo Gordejuela

Departamento de Álgebra

Facultad de Matemáticas

Universidad Complutense

28040-Madrid, Spain

jetayo@mat.ucm.es

Ernesto Martínez

Departamento de Matemáticas Fundamentales

Facultad de Ciencias, UNED

28040-Madrid, Spain

emartinez@mat.uned.es

The first author is partially supported by MTM2005-02865 and UCM910444 and the second one by MTM2005-01637. 